Estudios Románicos, Volumen 30, 2021, pp. 121-134

ISSN: 0210-4911

eISSN: 1989-614X

DOI: https://doi.org/10.6018/ER.471091

\title{
LA INTENSIFICACIÓN EN LOS CUENTOS POPULARES OCCITANOS
}

(Intensification in Occitan folktales)

\author{
Louise Esher* \\ CNRS (LLACAN, UMR 8135)
}

\begin{abstract}
This study offers an initial exploration of the intensification strategies attested in a written corpus of 162 Occitan folktales (north-east and south-west Lengadocian varieties). Although, overall, the pool of available resources corresponds to ordinary Occitan usage, the deployment of the strategies also reflects the particular conditions surrounding the storytelling and (cultural) transmission of folktales. Intensification is used to strengthen both the audience's immersion in the narrative, and the cultural and social cohesion of the community in which the stories are told.
\end{abstract}

Keywords: Occitan; Oral literature; Intensification; Visualisation; Collective memory; Cultural transmission.

Resumen: Este estudio plantea un análisis preliminar de las estrategias de intensificación constatadas en un corpus escrito de 162 cuentos populares occitanos (variedades languedocianas nororientales y sudoccidentales). Aunque, en términos generales, el acervo de recursos disponibles se corresponde con el uso corriente del occitano, la utilización de esos recursos también refleja las circunstancias específicas que condicionan la narración y transmisión (cultural) de los cuentos. La intensificación se utiliza para reforzar tanto la inmersión del público en la narración como la cohesión cultural y social de la comunidad donde se relatan los cuentos.

Palabras clave: Occitano; Literatura oral; Intensificación; Visualización; Memoria colectiva; Transmisión cultural.

* Dirección para correspondencia: Louise Esher. CNRS LLACAN (Langage, Langues et Cultures d'Afrique, UMR 8135). 7 rue Guy Môquet. 94801 Villejuif. France (louise.esher@cnrs.fr) 


\section{Introducción ${ }^{1}$}

Los cuentos populares son narraciones de tradición oral para las cuales el folclore cataloga motivos abundantes y recurrentes y establece una clasificación universal (Aarne 1961, Uther 2004). En el contexto occitano, hasta mediados del siglo XX, cuando la transmisión oral de la lengua se redujo drásticamente, los cuentos populares formaban parte de las actividades tradicionales de ocio en las comunidades y se destinaban a todos los públicos (esto es, a los adultos tanto o más que a los niños).

En una situación narrativa tradicional, la función primera de los cuentos populares es el espectáculo. La narración ha de ofrecer una distracción que capte y mantenga el interés del público, mediante la escenificación de elementos fantásticos o humorísticos, invitándolo a despojarse de su incredulidad y a sumergirse en el cuento, por muy inverosímil que este sea. Además, en su calidad de artefacto cultural, el cuento desempeña otra función fundamental, a saber, contribuir a crear y a preservar la identidad de la comunidad, su memoria colectiva y su cohesión social. Como todos los conocimientos culturales transmitidos por aprendizaje iterativo, los cuentos están sujetos a la presión evolutiva: no basta con enunciarlos para que se transmitan, sino que alguien debe recordarlos y considerar que merece la pena relatarlos de nuevo (Norenzayan et al. 2006).

El corpus de textos que fundamenta este estudio se enmarca en un movimiento destinado a la recopilación, la transcripción y la publicación de cuentos con el objetivo de salvaguardar el patrimonio inmaterial (cultural y lingüístico) que representan. Agrupa 162 textos: los 109 Secrèts de las Bèstias (Bèst., Bru 2014), recogidos por el profesor y escritor André Lagarde en los años 50 y 60 en el Pays d'Olme y el Quercorb (en las faldas pirenaicas, en los departamentos de Ariège y Aude), y los 53 Contes d'Aubrac (Aub., Bru; Eygun 2019), recopilados por la etnóloga Marie-Louise Ténèze en los años 60 en la región de Aubrac (al sur del macizo Central; departamentos de Aveyron, Cantal y Lozère). Según la clasificación tradicional de los dialectos occitanos, las variedades lingüísticas presentes en la selección de cuentos pertenecen al grupo languedociano, aunque las de la región de Aubrac lindan con el grupo auvernés (Alibèrt 1976, Oliviéri; Sauzet 2016) ${ }^{2}$.

En este estudio, se analiza los recursos de intensificación utilizados en los cuentos del corpus. Aunque, en términos generales, el abanico de procedimientos identificados se corresponde con el uso habitual del occitano languedociano oral, también refleja la doble función interpersonal de los cuentos: la diversión y la cohesión social.

\section{Los recursos de intensificación en los cuentos occitanos}

En este apartado, se propone una tipología cualitativa de los recursos identificados en los cuentos estudiados, con ejemplos que ilustran su utilización característica. La

1 Doy las gracias a Xavier Bach, Paloma Carrión Valencia, Peter Clayburn y a los dos revisores anónimos por sus constructivas observaciones. La autoría asume la responsabilidad de todo eventual error u omisión.

2 Por esta razón, los lectores podrían observar diferencias de forma entre las variedades estudiadas en este artículo y otras variedades del occitano. 
clasificación, inspirada en la propuesta de Romero (2017) relativa al caso francés, se organiza según los niveles de estructura lingüística y la forma de las construcciones. Han sido necesarias adaptaciones de categoría, pues el acervo de recursos del occitano no se corresponde exactamente con el del francés, ya sea debido a diferencias sintácticas o a la ausencia de algunas figuras retóricas en el corpus.

\subsection{Prefijos, sufijos y morfología evaluativa}

A diferencia de otras lenguas románicas, el occitano no utiliza el sufijo superlativo derivado del latín -ISSIMUM. Por otro lado, cuenta con pocos prefijos de intensificación, cuyo uso se circunscribe al registro formal o culto, que no es propio de los cuentos (ej. subrebèl 'hermosísimo', de subre + bèl 'hermoso, grande' (Alibèrt 1976: 56)).

Más importante es el papel de la morfología evaluativa. Como demuestra Bach (2020), los diferentes sufijos aumentativos del occitano (-às, -aràs, -atàs, Alibèrt 1976: 364-365) no denotan meramente dimensiones superiores o cantidades importantes, sino un atributo que se considera excesivo en el contexto específico del discurso: por ejemplo, la forma ostalàs (ostal 'casa' + às 'aumentativo') se emplea para describir una casa que, sin ser necesariamente muy grande, tiene un tamaño desmesurado para sus pocos ocupantes. Así, el sufijo aumentativo sirve para intensificar el atributo en cuestión, oponiéndose desde el punto de vista estructural al sufijo diminutivo, cuya función reside en atenuar (y que, al igual que otros muchos recursos de atenuación, originalmente era utilizado para indicar «poca cantidad»o «de pequeño tamaño» (Margerie 2007, Esher 2020).

Así, en los cuentos, el aumentativo denota un atributo excesivo o desproporcionado; a continuación se expone, a título de ejemplo, la reacción de un carbonero cuando un oso se adentra en su cabaña para guarecerse del frío invernal y ve cómo la escarcha que cubre el pelo del animal se derrite con calor de la chimenea:

l'aiga rajava e fasiá un bèlh lhacàs al mièg de la cabana. Aquel animalàs me negarà tot l'ostal, se pensava le carbonièr. (Bèst., 80) 'El agua chorreaba y formaba un gran lago+AUG en medio de la cabaña. -Aquel animal+AUG me anegará toda la casa- pensaba el carbonero'.

El oso, un animal de gran tamaño, ocupa demasiado espacio dentro de la pequeña cabaña del desdichado hombre; además, la abundante agua que esparce en el interior crea un charco, desmesurado por definición y cuya presencia en cualquier vivienda es, a todas luces, un inconveniente. Cabe señalar, asimismo, varios intensificadores: además del aumentativo, se recurre a la modificación mediante bèlh 'grande' y tot 'todo', y a los términos hiperbólicos lhac 'lago' y negar 'anegar' para describir un charco, que esencialmente es pequeño y poco profundo.

En muchos casos, la desmesura que denota el aumentativo hace referencia a un ente sobrenatural. Por ejemplo, las formas omenàs (òme 'hombre' + às, Bèst., 118) y femnassa (femna 'mujer' + assa, Bèst., 118) sirven para describir a un gigante y su mujer; manassa (man 'mano' + assa; Bèst., 118) designa la mano del gigante, tan desmesurada como él; y 
auselhàs (ausèlh 'pájaro' + às, Bèst., 231) indica un águila lo bastante grande como para transportar al protagonista humano, algo que sería imposible en el mundo real ${ }^{3}$. En otros casos, refleja un error de categoría o cierta inadecuación respecto del contexto, por ejemplo en el cuento de un herrero que ve a otros aldeanos pasar mientras trabaja:

corrisson darrèr un gossàs en cridant : Al lhop! Al lhop! Arrestatz-le!

Un lhoparràs, de fèt, es davalat del bòsc.

'corren detrás de un perro+AUG, gritando: ¡Al lobo! ¡Al lobo! ¡Detenedlo!

Un lobo+AUG, de hecho, ha bajado del bosque.'

Aquí, aunque el referente de gossàs se parece a un perro, en realidad se trata de un cánido salvaje, es decir, un lobo. Además, en el folclore, el lobo suele ser considerado peligroso, por lo que su presencia es inapropiada en el contexto del pueblo: los aldeanos se refieren al lobo con el término básico lhop, mientras que la narración emplea el aumentativo lhoparràs (lhop 'lobo' + aràs) para indicar que un lobo no tiene cabida en un pueblo.

\subsection{Palabras compuestas, unidades fraseológicas y repetición}

En el corpus de cuentos, las palabras compuestas que expresan intensificación se dividen por lo general en dos categorías. La primera, productiva, consiste en la reduplicación total de adjetivos: vièlha vièlha 'viejísima' (Bèst., 197), pichon pichon 'pequeñísimo' (Bèst., 116). La segunda recurre a locuciones que Romero (2017) clasifica como syntagmes variés de paires coordonnées: en occitano, la cuasi fijación de estas combinaciones de palabras (chut e mut 'callado', Bèst. 300; fòrt e mòrt 'enfáticamente, fuertemente', Bèst., 88) - las cuales, aunque parecen unidades coordinadas, no son construcciones composicionales en sentido estricto y no admiten la modificación de sus elementos constitutivos - nos permite considerarlas también compuestos lexicalizados.

En el caso de los verbos, existe una construcción fija "presente.3sG + que + futuro.2sG" (Alibèrt 1976: 349), que expresa el aspecto durativo e iterativo, generalmente para indicar un empeño constante y sostenido ${ }^{4}$. En los cuentos, se utiliza principalmente con verbos de desplazamiento para hacer referencia a un viaje de larga duración, por ej. marcha que marcharàs (Bèst., 164), camina que caminaràs, (Bèst., 207) —literalmente, 'camina que caminarás' - y con verbos que denotan un esfuerzo físico, en alusión a una tarea incesante y inacabable, como en la descripción del trabajo del herrero:

N'es atal tot le lhambre del jorn. Bufa que bufaràs, truca que trucaràs (Bèst., 104) 'Está así todo el día: sopla, que sopla; golpea, que golpea'.

3 En determinadas variedades, la forma aucelàs se ha lexicalizado con el nombre genérico "ave rapaz". En el contexto de este cuento popular, esa interpretación es poco probable porque en las tres referencias anteriores al ave en cuestión se ha utilizado el término específico agla 'águila'.

4 Otro análisis de esta construcción es posible, a saber, "imperativo.2sg + que + futuro.2sg", como expresión dirigida de manera imaginaria al protagonista. 
No cabe confundir tales construcciones específicas con la repetición: otro recurso que, con iconicidad discursiva, refleja la idea de insistencia o larga duración. La repetición se emplea tanto con sintagmas como con palabras simples, y permite emplazar más de dos iteraciones.

La poleta blanca se plorava, se plorava! (Aub., 259)

'La gallinita blanca lloraba, lloraba'

Alara se metèc a lhavassar e granissar, a lhavassar e granissar, a lhavassar e granissar... E aquò quatre jorns de reng (Bèst., 304)

'Entonces se puso a diluviar y granizar, a diluviar y granizar, a diluviar y granizar... Y así durante cuatro días seguidos'

seguèron lo fial, totjorn lo fial, totjorn lo fial, e naturalament, lo fial los menèt a l'ostal roge (Aub., 229)

'siguieron la cuerda, siempre la cuerda, siempre la cuerda, y naturalmente, la cuerda los condujo a la casa roja'

\subsection{Modificación adjetival, modificación adverbial y cuantificación}

En los cuentos, la intensificación mediante adjetivos y adverbios lexicales es secundaria. La intensificación adjetival se utiliza esencialmente con una cantidad limitada de adjetivos calificativos con el significado básico de atractivo, agradable o de gran tamaño: bèl ('hermoso, grande'), bon ('bueno'), brave ('bueno, amable'), gròs ('grande, grueso'), grand ('grande'). Cuando se usan como intensificador, estos recursos denotan que el sustantivo que modifican representa un modelo dentro de su categoría: un bèl borrèc 'un buen borrego (sano, desarrollado)' (Bèst., 82); un bon còp de martèl 'un buen golpe dado con el martillo (fuerte, preciso)' (Aub., 245); un brave miralh 'un buen espejo (grande, de calidad)' (Aub., 103); un gròs pauruc 'un miedoso absoluto' (Bèst., 33); grand rambalh 'gran bullicio' (Bèst., 186).

En cuanto a la intensificación adverbial, resulta curioso constatar la ausencia del adverbio común bravament 'mucho, bien': los cuantificadores son el recurso más frecuente. En los cuentos de Ariège y Aude, se emplea plan 'muy, bastante' y fòrça 'mucho': som plan malurós 'soy muy infeliz' (Bèst., 187); plan onèstament 'muy honestamente' (Bèst., 198); aquò's plan vertat 'eso es totalmente cierto' (Bèst., 76); me rendretz plan servici 'me seréis de gran ayuda' (Bèst., 188); èran fòrça malcorats 'estaban muy decepcionados' (Bèst., 185); I fasquèc fòrça compliments 'Le hizo muchos cumplidos' (Bèst., 203). En los de Aubrac, se emplea bièn 'muy, bastante': soi bièn content 'estoy muy contento' (Aub., 310); bièn luènh 'muy lejos' (Aub., 197); i aviá biènssas de pomas 'había muchas manzanas' (Aub., 229). Encontramos también ben 'efectivamente': ela, n'aviá ben vist un 'ella sí que había visto uno' (Bèst., 161), y fòrt 'fuerte': fòrt plan mesa 'muy bien vestida' (Bèst., 86), y, en ambas regiones, tot 'todo': una quilha tota redonda, tota lisa, tota prèsta 'un bolo todo redondo, todo liso, todo listo' (Bèst., 197); l'ostal tot frig, las pòrtas totas 
dubèrtas 'la casa toda fría, las puertas todas abiertas' (Aub., 233); tot doçament butèc le lhèit 'muy ligeramente empujó la cama' (Bèst., 179).

También se encuentran algunos cuantificadores lexicales, principalmente un fum de monde 'una multitud de gente' (Bèst., 199). El caso de un burro maravilloso que caga monedas de oro nos brinda varios ejemplos:

"Auràs qu'a i dire "Ase caga-denièr, caga-me'n un plen panièr», e de jos la coga i tombarà un flòc de peças d'aur" [...] L'ase lhèva la coga e una patracada de pèças redòlan sul pasiment (Bèst., 164-165).

- Solo tendrás que decirle: «Burro cagadinero, cágame de eso [=dinero] una cesta entera», - y debajo de su rabo caerán un montón de monedas de oro. [...] El burro levanta el rabo, y caen al suelo adoquinado una multitud de monedas'.

El contexto permite aclarar que «la cesta» no hace referencia a un objeto específico ni concreto: se trata de un cuantificador que expresa una noción de cantidad (y que permite tener rima), intensificado por el adjetivo plen 'lleno'; las monedas, que no necesitan recipiente alguno, caen en un número indefinido pero significativo (un flòc 'un montón', una patracada 'una multitud').

\subsection{Expresiones de modo y consecuencia: tant y talament}

Cabe señalar la escasa frecuencia con que se usa la modificación mediante adjetivos o adverbios lexicales en los cuentos. Por el contrario, son frecuentes las expresiones de modo o de consecuencia que permiten precisar una manifestación visible, tangible o sensorial de la intensidad.

Una construcción recurrente precisa una unidad de gran tamaño que califica una acción: caminava a bèlhas garradas 'caminaba a grandes zancadas' (Bèst., 137); a bèlhas urpadas grimpèc per las brancas amont 'a grandes zarpazos trepó por las ramas' (Bèst., 181); enfornava a bèlhis culherats 'engullía a grandes cucharadas' (Bèst., 218). Existe un evidente paralelismo estructural entre esta construcción y la que especifica que una acción se realiza con la mayor intensidad posible y ocupando por completo el espacio disponible: niflar a plenas nasicas 'olfatear a plenas narinas' (Bèst., 118); l'aiga rajariá pertot a plen canèlh 'el agua chorrearía por todas partes a pleno caño' (Bèst., 179); bufava a plenis palmons 'soplaba a pleno pulmón' (Bèst., 199).

En otras expresiones se precisa una consecuencia, ya sea real (introducida por la conjunción que) o hipotética (introducida por la preposición $a$ ), que necesariamente es inherente y cuantifiable: sufrir una herida que tardará ocho días en sanar, hacer que los fuertes ronquidos resuenen en todos los rincones de una casa, o hablar con un acento que provoca dolor físico en el interlocutor:

le Parisièn arriba, blanc coma un pòrre, mas fòrt plan atrencat, plan lhifrat e parlant ponchut a vos curar les èlhs (Bèst., 208) 
'llega el parisino, blanco como un puerro, pero muy bien vestido, todo acicalado y hablando de un «afilado» [=francés, o con acento francés, no occitano] que te raspaba los ojos'

te m'i fica una martelhejada sus la lhenga que de vèit jorns posquèc pas parlar (Bèst., 98) 'así que le propina tal martillazo en la lengua que durante ocho días no pudo hablar'

s'endurmisquèc boca badada que les ronquets fasián restrunhir tot l'ostal (Bèst., 108) 'se adormeció con la boca abierta y los ronquidos hacían retumbar toda la casa'

Las formas tan 'tan', tal 'tal' y talament 'tanto' están casi sistemáticamente asociadas a la expresión de consecuencias fictícias o reales:

tant adreit de sas mans qu'auriá fèit les èlhs a-n un gat (Bèst., 197)

'tan hábil con las manos que le habría hecho los ojos a un gato'

se fasquèc lèu una tala renommada que le venián quèrre de pertot (Bèst., 185)

'enseguida se hizo un renombre tal que lo venían a buscar de todas partes'

lo poiretz pas manjar qu'es talament dur (Aub., 265)

'no podréis comerlo de lo duro que está'

Por hiperbólicas que sean estas consecuencias en muchos casos, siempre evocan imágenes concretas y específicas.

\subsection{Superlativos}

A falta de superlativos sintéticos en occitano, el superlativo se suele formar con construcciones analíticas que asocian el artículo definido y el adverbio pus 'más' (le pus polit porquet, 'el lechón más bonito', Bèst., 191). Sin embargo, en los cuentos, el superlativo se expresa generalmente de manera más indirecta, afirmándose que no existe ningún elemento de comparación. Como no cabe identificar, imaginar o nombrar ese elemento, en realidad la entidad comparada no tiene parangón, de manera que constituye el summum (Romero 2015).

esperdigalhat coma digús (Bèst., 197)

'espabilado como nadie'

una crostada daurada res de pus polit (Bèst., 30)

'una crostada dorada más bonita que nada'

fringaire e calinhaire que se pòt pas dire (Bèst., 104)

'[tan] amoroso y enamorado que no se puede describir con palabras' 
En determinados casos, la exclusión explícita no se aplica solo al momento presente, sino con una permanencia absoluta:

unis caulets polits coma jamès pus (Bèst., 86)

'unos repollos bonitos como nunca más [se verían]'

unas nòças coma jamès se n'èra vist. E coma jamès non se’n virà (Bèst., 103) 'una boda como nunca se ha visto. Y como nunca se verá'

Esta estrategia, consistente en reforzar el superlativo y presentarlo como válido en todos los universos posibles, se emplea también con la forma analítica del superlativo:

la rauba pus meravilhosa que se’n pòsca somiar (Bèst., 89)

'el vestido más maravilloso con que se puede soñar'

Por último, también existen ejemplos de intensificación donde se recurre a la comparación (pus...que... 'más... que...') para expresar que algo supera o constituye el prototipo de una cualidad determinada, o de una noción propiamente dicha, esto es, que no tiene parangón (Romero 2015):

I vegèc la figura afrosa d'una vièlha,... pus rupada que poma cuèita (Bèst., 101)

'Allí vio la espantosa cara de una vieja,... más arrugada que una manzana cocida'

èra mès que content (Bèst., 123)

'estaba más que contento'

\subsection{Símiles y comparaciones estereotipados}

Los símiles permiten evocar una imagen precisa y vívida sin necesidad de hacer una descripción detallada, mediante el establecimiento de una comparación con cierta realidad concreta en la comunidad (Roberts; Kreuz 1994, García-Paje 2008, Mpouli; Ganascia 2017). Por definición, este recurso se basa en conocimientos culturales comunes, de manera que contribuye a la función de cohesión social ${ }^{5}$. Así, muchos símiles evocan un referente religioso: este puede ser el universo moral y doctrinal del cristianismo (el pecado reprobable, el diablo que representa el mal, las bendiciones cristianas intolerables para el diablo), o los objetos materiales de la liturgia (el cáliz de la comunión, la pila de agua bendita):

5 Cabe señalar que los referentes culturales no han de ser distintivos de una comunidad específica para cumplir con esa función. Por ejemplo: aunque las tradiciones cristianas trascienden las zonas geográficas en las que se recopiló el corpus de cuentos populares estudiado en este artículo (y, por ende, no caracterizan a las comunidades de esas zonas), tienen suma pertinencia para la cohesión social debido al arraigo de las prácticas religiosas en el entorno cotidiano de los miembros de la comunidad, ya sea en los edificios, los bienes, el clero, el culto colectivo, los festivales o los ritos de iniciación. 
La segonda èra lèda coma le pecat (Bèst., 30)

'La segunda [hija] era fea como el pecado'

voliá que l'ostal fosquèssa net coma un calici (Bèst., 57)

'quería que la casa estuviese limpia como un cáliz'

Fiula un bocin... e l'arpalhand de sautar coma un diable dins un aiga-senhadièr (Bèst., 51)

'Silba un poquito... y el hombretón se pone a brincar como un diablo en una pila de agua bendita'

Otros símiles reflejan el entorno natural y artificial de la comunidad, entre otros la aparencia de los árboles, los edificios y los animales más conocidos, o el comportamiento de ciertos animales:

A la nèit, montèc al cèl una lhuna redonda coma una ròda de molin (Bèst., 51)

'Al anochecer, apareció en el cielo una luna redonda como una rueda de molino'

Èra naut coma una torrassa, espatlut coma un buòu, garrut coma un avet (Bèst., 106)

'Era alto como una torre+AUG, ancho de hombros como un buey, vigoroso como un abeto'

Lèstes coma unis esquiròls, les gojatons davalhèron (Bèst., 119)

'Ágiles como ardillas, los chiquillos bajaron'

Algunos conjugan varios marcos de referencia. Por ejemplo, la interpretación de una princèssa polida coma un solelh de Pascas 'una princesa hermosa como un sol de Pascua' (Bèst., 92) precisa el conocimiento del calendario cristiano, para situar la Pascua en la primavera, así como del clima y del calendario agrícola, para comprender cuánto agrada ver el sol en esa época del año: solo aunando ambos marcos de referencia es posible imaginar el efecto que provoca la belleza de la princesa.

Los símiles también sirven de pretexto para explicitar las costumbres y creencias de la comunidad:

L'Ors èra coma Sant Tomàs: per tant asseguradas que sián las paraulas, s'i voliá pas fisar - caliá que vegèsse! (Bèst., 5)

'El Oso era como Santo Tomàs: por ciertas que fueran las palabras, no quería fiarse de ellas, ¡tenía que verlo!'

A! lo paure òme sufrissiá, pense ben, sangava coma un boc, coma un pòrc quand òm lo sanga; sabètz ben cossí aquò-z-es dins los ostals, òm tua cadun un pòrc e l'òm lo sanga, aquò sanga. E ben guel sangava coma un pòrc. (Aub., 171)

¡Ay! El pobre hombre sufría, piensa bien; sangraba como un macho cabrío, como un cerdo cuando lo desangramos; sabéis muy bien lo que sucede en las casas, matamos cada uno un cerdo y lo desangramos, se desangra. Pues él sangraba como un cerdo.'. 
Del temps que vos parli, i aviá encara un rictor dins la parròquia de Vilhac. Ara, fa qualque lhuna que la glèisa es tampada e que s'i ditz pas pus messa le dimenge. Talament qu'es passat en reprovèrbi de dire "eishec coma le calici de Vilhac». (Bèst., 201)

'En la época de la que os hablo, todavía había un rector en la parroquia de Vilhac. Ahora, hace unas lunas que la iglesia está cerrada y que allí ya no se dice misa los domingos. Tanto es así, que hay un proverbio que dice "sec[ad]o como el cáliz de Vilhac".'

Las narraciones permiten rememorar el pasaje evangélico del apóstol Tomás (Juan 20: 24-29), que se niega a creer en la resurrección de Cristo sin verlo ni tocarlo, y la tradición profana de la matanza del cerdo, según la cual las familias engordan un cerdo durante el verano y el otoño para sacrificarlo y tener provisiones para el invierno. En el último ejemplo, la narración evoca un proverbio local y explica su etimología, relacionándolo con el conocimiento del contexto local (la iglesia de Vilhac se encuentra abandonada). El llamativo contraste entre la situación real y la que presenta el cuento permite establecer la supuesta antigüedad de los hechos narrados, mediante la lítote de qualque lhuna 'alguna luna' con el significado de 'mucho tiempo'.

Aunque, en su mayoría, los símiles suponen asociaciones convencionales entre entes y atributos (princesas hermosas, gigantes de gran tamaño), en algunos casos permiten recalcar una asociación opuesta a la esperada; por ejemplo, el huevo del que sale un maravilloso pollo minúsculo llamado Mièg-Polet 'Medio-Pollo': a diferencia de los huevos prototípicos (redondos), este es plano. Otro ejemplo lleva a un engaño cómico: en un año de buena vendimia, el rector pide que cada aldeano le obsequie con un poco de vino, pero la mayoría echa agua al barril del rector en vez de vino; así, cuando lo abre, el vino resulta muy diluido y prácticamente incoloro.

allòc d'èsser redond coma son totis, aquel uòu èra plat: plat coma la borsa d'un paure òme

'en lugar de ser redondo como son todos, ese huevo era plano: plano como el monedero de un hombre pobre' (Bèst., 147)

le vegèc pishar gaireben tan clar coma la font de la placeta (Bèst., 283)

'lo vio [el líquido] chorrear casi tan claro como la fuente de la plazoleta'

\subsection{Lítote}

Se trata de una figura retórica que, aunque parece atenuar, en realidad conduce a una interpretación de acusada intensificación (Jaubert 2008). En los cuentos se usa con frecuencia, en el contexto de la descripción detallada de una situación extrema: por ejemplo, la hambruna que padece una familia desde largo tiempo, o el aspecto repugnante de hombres ahorcados (que, al estar muertos, no pueden responder al protagonista cuando los interpela): 
A la bòrda de Pica-Talent - que le nom sol ba ditz - vivián pas dins l'abondància (Bèst., 127)

'En la granja de Hambre-Punzante — su mero nombre lo dice todo- no vivían en la abundancia'

Èran pas polits a véser, podètz creire! les èlhs revirats, le còl estirat, la figura grimaçuda ande una lhenga de tres pams. "E ben, les amics? Ètz pas plan parlaires..." (Bèst., 122)

'No eran bonitos de ver -ipodéis creerlo! - los ojos en blanco, el cuello estirado, la cara torcida con una lengua de tres palmos. — iY bien, amigos? ¡No estáis muy habladores!'.

En muchos casos, la lítote se ve acompañada por los intensificadores plan, bièn: èra pas bièn riche 'no era muy rico' = 'era demasiado pobre' (Aub., 299); passava per èsser pas plan degordit 'pasaba por no ser muy espabilado' = 'parecía muy simplón' (Bèst., 135). Se asocian también con una morfología evaluativa, aumentando así su efecto: aquel enfant èra pas bièn intelligentàs 'ese joven no era muy inteligente+AUG' $(A u b ., 197)$ describe a un joven cuyas tonterías serán el tema central de un cuento humorístico.

\subsection{Interjecciones, exclamaciones y visualización}

Varias narraciones emplean interjecciones o exclamaciones para resaltar un ente, un atributo o un evento importante. Mediante esos recursos, se genera la inmersión narrativa del público (Allan 2018), que puede adoptar diversas perspectivas.

En las interjecciones invariables, la narración adopta la perspectiva del protagonista y expresa sus reacciones de ánimo por lo general el asombro en situaciones que no se

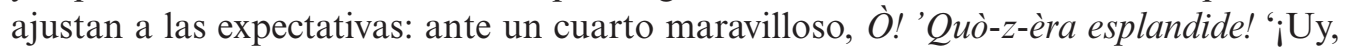
eso era espléndido!' (Aub., 309); ante una tarea imposible, ela coneissiá pas res pichaire 'ella no conocía nada, jay, qué lástima!' (Aub., 310); y ante una aparencia engañosa, Aquel elixir, pardí! èra un endurmitòri 'Ese elixir, ipor Dios! Era un somnífero' (Bèst., 162).

Mediante palabras exclamativas (quin 'cuál', cossí 'cómo'), la narración invita al público a percibir de manera directa (aunque imaginaria) el grado de una cualidad o la intensidad de una acción. En algunos casos, se trata de ponerse en la piel de los protagonistas: Quin espant '¡qué susto!' (Bèst., 168); gaitatz cossí lhusís 'mirad cómo brilla' (Bèst., 86). Más común todavía es la invitación explícita a la visualización, imaginándose como testigo directo; en estos casos, se recurre a un verbo de percepción en una oración condicional (ej. se vesiátz 'si vierais' o auriátz vist 'habríais visto'), con la premisa implícita de que "estuvierais presentes".

Bon Dieu, paures amics! Se vesiátz quin carnatge! (Bèst., 83)

‘Ay, Dios, pobres amigos! ¡Si vierais qué matanza!’ 
Auriátz vist aquelhas femnas, cossí èran contentas! (Bèst., 78)

'Habríais visto esas mujeres, ¡cuán felices estaban!'

Auriátz entenduda la dalha, cossí Jan la fasiá fiular. E per las sègas, auriátz vist le volam cossí le fasiá dançar (Bèst., 128)

'Habríais oído la guadaña, como Jan la hacía silbar. Y en la cosecha, habríais visto la segadera, como la hacía bailar'

Este procedimiento de visualización es ampliamente conocido en las narraciones destinadas a la interpretación oral, como la poesía épica clásica (Bakker 1993, Allan 2018) y el cantar de gesta medieval (Leverage 1999: 84-98). Según el análisis de Leverage, el cantar de gesta no solo anima al público a "imaginatively recreate, both the visual and the aural aspects of a particular scene" (1999: 92), sino que, mediante esa recreación sensorial, ayuda a grabar el episodio narrativo en la memoria (1999: 93). Así, en los cuentos, este recurso fomenta tanto la diversión como la memoria colectiva.

\subsection{Comentarios}

A continuación, figura una reflexión muy reveladora de una narradora que, al contar la historia de un rector cuya casa había sido presa de las llamas, evoca la versión que le había relatado otra narradora:

La Finon d'en Porcelh, quand nos contava aquel afar, disiá: "Le tirèron del lhèit rastumat coma un piòt qu'aurián debrembat a l'aste»... Mès per fèr rire, pr'aquò, n'i cal pas metre tròp! (Bèst., 27)

'La Finon d'en Porcelh, cuando nos contaba ese asunto, decía: «Lo sacaron de la cama chamuscado como un pavo olvidado en un asador...» - Pero para hacer reír, ¡no hay que exagerar!’

La narradora recuerda de manera muy clara el símil llamativo y cómico de la versión anterior, pero duda a la hora de integrarlo completamente en su versión personal, por no socavar ni la verosimilitud del cuento ni la inmersión del público. Así, opta por mencionarlo como otra referencia cultural común, incluyendo en el cuento el recuerdo de la tal Finon. Esta reflexión ilustra tanto la doble función del cuento, donde coexisten la distracción divertida y la memoria colectiva, como la presión evolutiva de la transmisión cultural, que prioriza los aspectos destacados (Barrett; Nyhof 2001), sin admitir excesiva inverosimilitud (Barrett et al. 2009).

\section{Conclusión}

Este análisis preliminar de los recursos de intensificación en un corpus de cuentos populares contribuye al conocimiento de la pragmática del occitano, que hasta la fecha no se ha descrito profusamente. El acervo que establece demuestra la variedad de re- 
cursos de intensificación presentes en los cuentos populares - morfológicos, lexicales, sintácticos y pragmáticos, o interpersonales-, muchos de los cuales tienen el efecto de facilitar la implicación del público, bien invitándolo explícitamente a la visualización, bien mediante detalles concretos o la evocación de conocimientos culturales comunes. Además, los ejemplos denotan una acusada tendencia a utilizar simultanéamente varios recursos (cuya investigación en profundidad trasciende el ámbito del presente estudio): los cuantificadores, la modificación y la repetición se emplean rara vez de manera aislada y suelen verse acompañados por formas de la morfología evaluativa, los símiles, la lítote y la expresión de modo y consecuencia.

\section{BIBLIOGRAFÍA}

AARNE, Antti (1961): The Types of the Folktale: A Classification and Bibliography, translated and enlarged by Stith Thompson. Helsinki: Suomalainen Tiedeakatemia.

ALIBÈRT, Loís (1976): Gramatica occitana segon los parlars lengadocians. Montpelhièr: Centre d'Estudis Occitans.

ALLAN, Rutger (2018): “Construal and immersion”, Peter Meineck; William Short; Jennifer Devereux (eds.). The Routledge Handbook of Classics and Cognitive Theory. Abingdon: Routledge.

BACH, Xavièr (2020): "Morfosemantica dels augmentatius en occitan del Lengadòc", Jean-François Courouau; David Fabié (eds.). Fidelitats e dissidéncias/ Fidélités et dissidences. Actes du XIIe congrès de l'Association Internationale d'Etudes Occitanes. Toulouse: Section française de l'Association internationale d'études occitanes, 115-120.

BAKKER, Egbert (1993): "Discourse and performance: Involvement, visualization and 'presence' in Homeric poetry". Classical Antiquity 12 (1): 1-29.

BARRETT, Justin; NYHOF, Melanie (2001): "Spreading of non-natural concepts: The role of intuitive conceptual structures in memory and transmission of cultural materials". Journal of Cognition and Culture, 1, 69-100.

BARRETT, Justin; BURDETT, Emily Reed; PORTER, Tenelle (2009): "Counterintuitiveness in Folktales: Finding the Cognitive Optimum". Journal of Cognition and Culture. 9: 271-287.

BRU, Josiane (2014): Andrieu Lagarda: Les Secrèts de las Bèstias. Pau: Letras d'Oc.

BRU, Josiane; EYGUN, Jean (2019): Contes d'Aubrac recueillis par Marie-Louise Ténèze. Pau: Letras d'Òc.

ESHER, Louise (2020): "Grammaticalisation et valeurs pragmatiques des expressions signifiant un peu en français et en occitan", Mercedes Banegas Saorin; Jean Sibille (eds.). Entre francisation et démarcation. Usages hérités et usages renaissantistes des langues régionales de France. Paris: L'Harmattan, 61-78. 
GARCIA-PAJE, Mario (2008): "La comparativa de intensidad: la función del estereotipo". VERBA. 35: 143-178.

JAUBERT, Anna (2008): "Dire et plus ou moins dire. Analyse pragmatique de l'euphémisme et de la litote". Langue française. 160(4), 105-116.

LEVERAGE, Paula (1999): The Chanson de Geste and Memory. Tesis doctoral, University of Toronto.

MARGERIE, Hélène (2007): "From downgrading to (over) intensifying: A pragmatic study of English and French", Istvan Kecskes; Laurence Horn (eds.). Explorations in pragmatics: Linguistic, cognitive, and intercultural aspects. Berlin: Mouton de Gruyter, 287-311.

MPOULI, Suzanne; GANASCIA, Jean-Gabriel (2017): "Another Facet of Literary Similes: A Study of Noun+Colour Term Adjectives". Corela [En ligne]. HS-21.

OLIVIERI, Michèle; SAUZET, Patrick (2016): "Southern Gallo-Romance (Occitan)", Adam Ledgeway; Martin Maiden (eds.). The Oxford Guide to the Romance Languages. Oxford: OUP, 319-349.

NORENZAYAN, Ara; ATRAN, Scott; FAULKNER, Jason; SCHALLER, Mark (2006): "Memory and mystery: the cultural selection of minimally counterintuitive narratives". Cognitive Science. 30: 531-553.

ROBERTS, Richard; KREUZ, Roger (1994): "Why Do People Use Figurative Language?". Psychological Science. 5(3),159-163.

ROMERO, Clara (2015): "Å̀ quoi compare-t-on pour intensifier ? Analyse du comparant dans les comparaisons d'intensité stéréotypées ou inventives", Krystyna Wróblewska-Pawlak; Anna Kieliszcyk (eds.). L'intensification et ses différents aspects. Varsovie: Presses de l'Université de Varsovie, 133-152.

(2017): L'intensité et son expression en français. Paris: Ophrys.

UTHER, Hans-Jörg (2004): The Types of International Folktales: A Classification and Bibliography. Based on the system of Antti Aarne and Stith Thompson. Helsinki: Suomalainen Tiedeakatemia.

\section{PERFIL ACADÉMICO Y PROFESIONAL}

Louise Esher trabaja de chargé de recherche en el CNRS desde 2016. Participa en el grupo de investigación LabEx EFL GL4 sobre la tipología y la flexión no canónica, en proyectos ANR de descripción del 'creciente linguístico' (zona de transición entre hablas occitanas y hablas de oïl) y en el proyecto Interreg POCTEFA LINGUATEC (desarrollo de recursos para la digitalización de las lenguas de los Pirineos). Sus campos de investigación se centran en la flexión verbal, la lingüística histórica y la lingüística románica, con un interés especial en las hablas occitanas.

Fecha de recepción: 26-03-21

Fecha de aceptación: 07-05-21 